\title{
A Framework for Multi-Participant Narratives Based on Multiplayer Game Interactions
}

\author{
Callum Spawforth and David E. Millard \\ University of Southampton, Southampton, United Kingdom, \\ cs14g13@soton.ac.uk
}

\begin{abstract}
Multi-participant Interactive Narratives have the potential for novel types of story and experiences, but there is no framework to show what is possible, and therefore no description of what types of multi-participant narrative could exist. In this paper, we attempt to build such a framework by first considering the core characteristics of interactions in multiplayer games, and then considering how those might be used to define different types of multi-participant narrative. Our framework is based on a systematic analysis of 56 interactions across 17 multiplayer games, resulting in 9 distinguishing characteristics. We then validate this framework by applying it to 3 novel multiplayer games, showing that it successfully captures the player interactions, although some higher level design decisions are missed. Finally, we demonstrate that novel premises for multi-participant narratives can be constructed from these characteristics. Our work provides a foundation for considering the types of multi-participant narrative that are possible.
\end{abstract}

Keywords: multi-participant narrative, multiplayer games, interactions

\section{Introduction}

The Interactive Digital Narrative (IDN) space has traditionally been focused on single-participant experiences[1-4], where a single reader interacts with a story system and has some agency over their experience of the story.

Narratives with multiple participants have the potential for interesting and novel types of story, but no clear approach to creating them exists, despite previous work in the area[5-7]. However, multiplayer video games are both widely studied, and applicable to the narrative space[8]. They support inter-player agency, where all participants collectively have agency over the experience. In a narrative context this raises interesting questions, such as to what extent participants have agency over each other's narratives, and how they might see the agency of others. The problem with answering these questions is that we have no framework that describes what types of multi-participant narrative might exist.

In this paper, we attempt to construct such a framework using a categorisation of the interactions between players in multiplayer games. We have undertaken our own categorisation because existing research in multiplayer game 
interactions takes a game design perspective, using concepts that are not necessarily applicable to narratives such as considering avatars or skills[9-11].

We seek to answer two key questions: what are the core characteristics of interactions in games, and how can we use those characteristics to describe possible novel multi-participant narratives?

The framework is developed through a systematic analysis of a sample of top-rated multiplayer games $(\mathrm{n}=17)$ across a variety of genres, and is validated by applying it to three video games that contain novel types of multiplayer experience and were not in the original sample. Finally, we create three story premises using the framework to demonstrate that by varying the characteristics within it we can inspire fundamentally different multi-participant narratives.

To our knowledge our work is the first attempt to define the range of possibilities for multi-participant narratives. In Section 2 we present a brief overview of existing work on interactions in digital games. Section 3 outlines our methodology for creating and validating the framework. Section 4 presents a description of the framework and provides an example of classifying the interactions from one of the sample games. Section 5 then presents the results of validating the framework against three games with novel approaches to multiplayer. Finally, in Section 6 we present three story premises inspired by the different characteristics within the framework, before concluding the paper in Section 7.

\section{Background: Analysing Multiplayer Games}

A previous approach to categorising multiplayer games is to model properties of the game. A simple model of multiplayer games consisting of rules, goals, props and tools was proposed by Zagal[12]. He identified six characteristics including "Social Interaction", "Cooperation and Competition" and "Synchronicity". While these could influence the overarching design of a narrative, their low fidelity prevents more detailed constructions.

Rocha[9] used a higher-fidelity approach, identifying a number of design patterns for cooperative mechanics. For example, the pattern "Complementarity", in which the abilities of players complement each other. Seif[13] extended this with further patterns, such as "Shared puzzles". While the essence of these patterns may apply in the narrative space, they assume concepts such as character abilities and manipulatable objects, which may not be true for narratives.

Reuter[10] developed a more applicable set of design patterns for collaborative multiplayer games. These are abstract, allowing them to be realised through a variety of different interaction mechanics. While patterns such as "Gathering Gate" are easily visualised in narrative, their abstract nature still leaves the challenge of implementation. Manninen[14] analyses interactions in more detail, proposing that interaction forms are "perceivable actions that act as manifestations of the user-user and user-environment interaction". His taxonomy of these enumerates different types of interaction, but it heavily focused on communication and doesn't identify how the nature of those interactions differs[15]. 
Our work focuses on modelling the differences between interactions at a higher fidelity than the earlier works, in order to understand how interactions may exist in the narrative space.

\section{Methodology}

The Systematic Analysis was an iterative coding analysis over the most popular games from Metacritic ${ }^{1}$. Metacritic was used due to the high per-game user rating count, quantity of games, and wide range of genres.

An initial sample list was created from the top 150 games of each of the 18 Metacritic genres. Non-multiplayer games were then removed, as were games with fewer ratings than the median of 39. The top game (sorted by user rating) from each genre was then selected to be analysed. However, the 'Party' genre was later removed as it primarily consisted of compilations of smaller games. This resulted in a sample set of 17 games, spread across 17 different genres with publication dates ranging from 1998 to 2013.

For each selected game, a single gamemode was chosen for analysis. This decision was based on the mode with the most information available (a particular problem with older games), typically the main or default gamemode.

A type of iterative coding was then performed on the chosen games. Interactions were identified using available information on the game, primarily video footage, articles, and reviews. During this process, some games were eliminated due to a lack of available information. When this occurred, the next highest rated game with sufficient information replaced it in the sample.

For our analysis we used a definition of interaction grounded in the works of Reuter[10] and Manninen[14], which state that interactions are "perceivable actions" with "perceivable visualisations".

For us, an interaction consists of two participants: an initiator and a recipient, and two parts: an action and an effect. An action is the command the initiator gives the system, such as "fire a bullet in this direction". The effect is the impact that action has on the game state, such as "injure this person and make a noise". The effect must be perceived by another player, in order for this to be an interaction[10].

An initial framework was created based on this definition, focusing on action and effect. As each interaction was identified, we attempted to classify it using the current framework. If an interaction could not be adequately described by the current set of characteristics, these were refined using the problematic interaction and other hypothetical edge cases to guide discussion. The refined characteristics were then reapplied against all identified interactions. This process continued until all identified interactions could be described by the refined characteristics. The identification of new interactions was then resumed.

This iterative process allowed for the creation of our framework and acted as a form of self-verification. However, we also wanted to test the framework

\footnotetext{
1 metacritic.com
} 
against games that were not in the sample set, and which were known for their unusual multiplayer mechanics.

To this end we applied the framework to three games considered to offer players a novel multiplayer experience, with the aim of discovering whether the framework adequately described the novel elements. The three games selected were Dark Souls, Journey and Dead by Daylight.

Our methodology is not intended to give an overall picture of interactions in multiplayer games. Although our sample is varied, we would not claim that it is representative. Rather we are using our selection criteria to ensure that the framework is based on the interactions of popular and well-known games, and see our contribution as the framework, rather than the classification itself.

\section{The Framework}

The games analysed are shown in Table 1. In total 56 interactions were identified from 17 games. From these interactions, 9 characteristics were identified and added to the framework, shown as a summary in Table 2 alongside examples from the sample set. Three characteristics address the interaction as a whole, while three focus on the initiator and another three on the recipient.

Table 1. Games classified during Framework Construction

\begin{tabular}{|l|c|l|l|c|}
\hline \multicolumn{1}{|c|}{ Name } & $\begin{array}{c}\text { Short } \\
\text { Code }\end{array}$ & Date & Gamemode & $\begin{array}{c}\text { Num. Found } \\
\text { Interactions }\end{array}$ \\
\hline Ratchet \& Clank: Up Your Arsenal & RC & 2004 & Siege & 4 \\
\hline Dragon Ball Z: Budokai Tenkaichi 3 & DBZ & 2005 & Versus & 2 \\
\hline The Last of Us & LU & 2013 & Survivors & 9 \\
\hline Counter-Strike & CS & 2000 & Bomb-defusal & 7 \\
\hline IL2-Sturmovik & IL2 & 2001 & Team-deathmatch & 3 \\
\hline Super Mario Advance 4 & IL2 & 2003 & Cooperative & 1 \\
\hline World of Goo & WG & 2008 & Cooperative & 4 \\
\hline Midnight Club 3: DUB Edition & MC3 & 2005 & Capture the Flag & 3 \\
\hline Starcraft & SC & 1998 & 2v2 Siege & 4 \\
\hline Mario and Luigi: Superstar Saga & ML & 2003 & Main Game & 1 \\
\hline Race 07: Official WTCC Game & R07 & 2007 & Race & 2 \\
\hline Greg Hastings Tournament Paintball & GHTP & 2005 & Elimination & 2 \\
\hline Advance Wars 2: Black Hole Rising & AW2 & 2003 & FFA Skirmish & 2 \\
\hline $\begin{array}{l}\text { James Bond 007: } \\
\text { Everything or Nothing }\end{array}$ & JB & 2003 & Cooperative & 3 \\
\hline Fire Emblem & FE & 2003 & Versus & 2 \\
\hline Toy Soldiers & TS & 2010 & Versus & 5 \\
\hline WWE Day of Reckoning & WWE & 2004 & Exhibition & 2 \\
\hline
\end{tabular}




\section{General Characteristics}

Likelihood. When a player takes an action, an interaction only occurs if the effect of that action is perceived by another player[10]. Likelihood is the chance that an interaction occurs. It is guaranteed if the recipient can notice the effect regardless of their current situation or state, such as a message that always appears when a given action occurs. An interaction is possible if the recipient must be in a particular situation or state to experience the effect, such as needing to be visit a box to see that an item has been taken.

Table 2. A summary of the characteristics identified in the 17 games analysed

\begin{tabular}{|c|c|c|c|}
\hline Characteristic & Value & Count & Example \\
\hline \multicolumn{4}{|c|}{ General Characteristics } \\
\hline \multirow{2}{*}{ Likelihood } & Guaranteed & 35 & LU: "Killing a player" \\
\hline & Possible & 21 & LU: "Emptying a Box" \\
\hline \multirow{2}{*}{ Туре } & Mechanical & 52 & CS: "Shooting and injuring a player" \\
\hline & Informational & 4 & WG: "Moving the cursor" \\
\hline \multirow{2}{*}{ Synchronicity } & Synchronous & 56 & LU:"Killing a player" \\
\hline & Asynchronous & 0 & No example classified \\
\hline \multicolumn{4}{|c|}{ Recipient Characteristics } \\
\hline \multirow{3}{*}{$\begin{array}{c}\text { Explicit } \\
\text { Awareness }\end{array}$} & Always & 10 & LU:"Healing an ally" \\
\hline & Possibly & 12 & TS: "Taking control of a unit" \\
\hline & Never & 34 & AW2: "Capturing a base" \\
\hline \multirow{3}{*}{$\begin{array}{l}\text { Deductive } \\
\text { Awareness }\end{array}$} & Always & 49 & FE: "Attack an enemy unit" \\
\hline & Possibly & 7 & TS: "Attacking using a unit" \\
\hline & Never & 0 & No example classified \\
\hline \multirow{3}{*}{$\begin{array}{c}\text { Initiator } \\
\text { Identifiability }\end{array}$} & Always & 36 & CS: "Killing a player" \\
\hline & Possibly & 20 & LU:"Emptying a box of items" \\
\hline & Never & 0 & No example classified \\
\hline \multicolumn{4}{|c|}{ Initiator Characteristics } \\
\hline \multirow{3}{*}{$\begin{array}{c}\text { Explicit } \\
\text { Feedback }\end{array}$} & Always & 8 & LU: "Shooting at and hitting a player" \\
\hline & Possibly & 8 & LU:"Emptying a box of items" \\
\hline & Never & 40 & TS: "Queuing up a unit for deployment" \\
\hline \multirow{3}{*}{$\begin{array}{l}\text { Deductive } \\
\text { Feedback }\end{array}$} & Always & 34 & WG:"Moving the shared view" \\
\hline & Possibly & 22 & CS: "Dropping a weapon on the ground" \\
\hline & Never & 0 & No example classified \\
\hline \multirow{3}{*}{$\begin{array}{c}\text { Recipient } \\
\text { Identifiability }\end{array}$} & Always & 42 & LU:"Healing an ally" \\
\hline & Possibly & 14 & LU:"Emptying a box of items" \\
\hline & Never & 0 & No example classified \\
\hline
\end{tabular}

Interaction type. An interaction can be Informational or Mechanical. Informational interactions only change the information available to the other player, or enable further interactions that are informational. One clear example from the unclassified game Team Fortress 2, is spraying a decal on a surface. Me- 
chanical interactions make more concrete alterations to the other player or their gameworld, directly impacting the other player's agency.

Synchronicity. Adapted for interactions from Zagal's game-wide definitions[12], a synchronous interaction requires that all interaction participants be participating in the game at the same time. For example, applying a medical kit to a player in The Last of Us. In contrast, in an asynchronous interaction at least one of the players may not be actively participating in the game. While no examples of this arose in the analysis, Multi-User Dungeons are known to have asynchronous elements[12].

\section{Recipient Characteristics}

Explicit Awareness. If the game explicitly informs the recipient that an effect was caused by another player, they are explicitly aware. This must always be extradiegetic[16], as it refers to the concept of a player. If this information is always perceivable, the recipient is always explicitly aware, for example a notification stating "Player X has scored a point". If the player may not be able to perceive this information, they are possibly explicitly aware, such as a message that only appears to players in a certain location. They may also never be explicitly aware.

Deductive Awareness. The recipient is deductively aware if it is possible to deduce that an effect was caused by a player from the game's rules. It may always possible to deduce an effect was caused by a player. For example, if a territory is captured, and only players are able to capture territories. However, it may only be possible to deduce in some situations, such as if a game has both players and environmental factors that can injure the recipient, and the recipient only knows that they have been injured. It is also possible that it may never be possible to deduce the source of the effect was a player.

Initiator Identifiability. Identifiability describes whether the recipient knows the identity of the player that has affected them. The initiator is always identifiable if the recipient always knows the player that caused the effect, for example if "[Initiator Name] has killed [Recipient Name]" always appears on the recipient's death. They are possibly identifiable if their ability to do depends upon their current situation or game state, e.g. they can observe who it is but are not guaranteed to. They may also never be able to identify the initiator.

\section{Initiator Characteristics}

Explicit Feedback. The counterpart to Explicit Awareness, the initiator receives explicit feedback if the game makes it explicit that they have affected a player with their action. Explicit feedback can either always, possibly or never occur in the same manner as Explicit Awareness. 
Deductive Feedback. The counterpart to Deductive Awareness, the initiator receives deductive feedback if they can deduce from the rules and information available that they've affected a player. Deductive Feedback can either always, possibly or never occur in the same manner as Deductive Awareness.

Recipient Identifiability. The counterpart to Initiator Identifiability, this characteristic addresses whether the initiator can identify the affected recipient, but otherwise functions identically.

\subsection{Communication, Bots, and Indirect Effects}

A number of aspects were removed from consideration during classification, as they added significant ambiguity when assigning values to the framework.

The presence of free-form communication, such as voice and text based chat channels, obfuscates Feedback, Awareness, Visibility and Identifiability. The information available purely within an interaction is overridden by allowing the player to inform others of their participation.

Similarly, many games contain game-playing agents ("bots") that attempt to imitate humans. These can make it hard to determine the value for a particular characteristic, due to the difficulty of differentiating between bots and players. An initiator may believe they have affected a player, when they have instead affected a bot.

Finally, secondary effects arising from the context of an interaction were not considered within the framework. For example, a player may defeat another player, in turn saving the life of a third player.

\subsection{Example Classification}

Due to size limitations we cannot show the full classification, but as an example

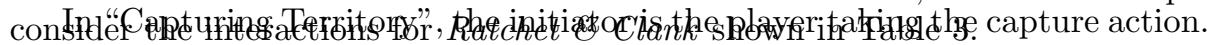
The recipient can be considered as any other player in the game. Capturing the area enables the initiator's team to revive at that location, making it a mechanical interaction. It is a guaranteed interaction, as it updates a persistent user-interface element. The recipient is always deductively aware, as only players can capture control points. The initiator identifiability is possible as it requires the recipient to be in the vicinity and to observe the capture. For the initiator, they can deduce that they've affected every player, as every player either gains or loses access to a control point. The initiator can identify every recipient, as everyone in the game is affected.

It can be seen from Table 3 that the collection of interactions that make up this game mode have different profiles. This demonstrates why it is important to classify individual interactions rather than the game as a whole, as the aggregate of the interactions fails to capture these interesting differences, and would tend to converge on a value of 'possibly' for most of the characteristics. Modelling the interactions increases complexity but maintains the fidelity of the analysis, and allows for more meaningful comparisons. 
Table 3. Example Interactions from "Ratchet \& Clank: Up Your Arsenal", Siege Mode

\begin{tabular}{|l|c|c|c|c|}
\hline Interaction Name & $\begin{array}{c}\text { Shooting and } \\
\text { hitting a } \\
\text { named player }\end{array}$ & $\begin{array}{c}\text { Shooting and } \\
\text { missing a } \\
\text { named player }\end{array}$ & $\begin{array}{c}\text { Killing } \\
\text { a player }\end{array}$ & $\begin{array}{c}\text { Capturing } \\
\text { Territory }\end{array}$ \\
\hline Likelihood & Guaranteed & Possible & Guaranteed & Guaranteed \\
\hline Type & Mechanical & Informational & Mechanical & Mechanical \\
\hline Synchronicity & Sync. & Sync. & Sync. & Sync. \\
\hline Explicit Awareness & Possibly & Never & Always & Possibly \\
\hline Deductive Awareness & Possibly & Possibly & Possibly & Always \\
\hline Initiator Identifiability & Possibly & Possibly & Always & Possibly \\
\hline Explicit Feedback & Possibly & Possibly & Always & Possibly \\
\hline Deductive Feedback & Possibly & Possibly & Possibly & Always \\
\hline Recipient Identifiability & Always & Possibly & Always & Always \\
\hline
\end{tabular}

\section{Framework Validation}

Having produced the framework and classified our sample set of games, we wanted to validate it by applying it to games that were outside the sample, but are known for their interesting approach to multiplayer. Each forms a small case study, that helps reveal the value and limitations of the framework.

\subsection{Case Study 1: Dark Souls}

Dark Souls is an action RPG, developed by FromSoftware and published by Namco in 2011 on a variety of 7th generation platforms. Dark Souls has a novel multiplayer system in which each player plays the game within their own version of the game's world. Various interactions exist that allow players to interact with the worlds of others, with "Signs" being one of the most prevalent.

"Signs" are runic symbols placed on the ground by players that have a chance to appear in other players' worlds. Signs allow the player to summon another player to their world, view a message, or invade the other player's world. The Gravelord Soul Sign, a type of invasion sign, also creates powerful opponents in the other player's world. The interesting interactions associated with these signs is when the initiator places the sign, which then appears in the recipient's world. Placing a sign has unusual characteristics according to our framework. Two types of sign (Message and Gravelord) are asynchronous. When placing all of these signs, feedback never occurs nor is the recipient identifiable.

Many interactions within Dark Souls are either a secondary result of the player's actions or are unintentionally triggered. For example, each death has a chance of leaving a "bloodstain" in the worlds of other players, which they can use to glean information about potentially dangerous areas. These passive interactions were also unusual in our original sample; the initiator in these cases is not identifiable nor is the recipient, and no feedback occurs. These characteristic values are unique to Dark Souls out of the twenty games classified in total, demonstrating a certain novelty in the games approach to multiplayer. 


\subsection{Case Study 2: Journey}

Journey is an exploratory adventure game, developed by thatgamecompany and published by Sony Computer Entertainment in 2012 on 7th generation platforms. Its multiplayer system is notable in that it connects strangers, and limits their ability to communicate to in-game actions.

In Journey, players communicate in a limited sense by briefly creating a variable-size sphere above their avatar. Energy is restored to the other player if they touch the sphere. We categorised this as two interactions, one when the sphere is only seen and another when energy is restored. Both are synchronous with recipient and initiator identifiable. However, while the first is informational, possible interaction with possibly deducible awareness and feedback, the energy interaction is mechanical and guaranteed with always deducible awareness and feedback. However, in practice both interactions are frequently used to provide information to the other player. This suggests that in the second interaction the mechanical function is hiding the equally significant informational one.

A novel mechanic within Journey is that each player's energy is recharged when players are in very close proximity. Triggering this was categorised as a guaranteed, mechanical, synchronous, always identifiable interaction with always deductive awareness and feedback, this is an identical classification to interactions in both IL-2 Sturmovik and World of Goo. The novelty stems from Journey's design, which uses this subtle interaction to encourage a collaborative experience.

The most novel element of Journey is the way in which players are matched without explicit effort. As a player progresses, another player will simply appear, and many players fail to realise the newcomer is another player. Despite the significance of this matchmaking it cannot be considered an interaction (no triggering action) and therefore does not appear in our framework. This suggests that further study of the way in which players are matched in games is needed.

\subsection{Case Study 3: Dead by Daylight}

Dead by Daylight is a survival horror game, developed by Behavior Interactive and published by Starbreeze Studios in 2016 for 8th generation platforms. It is notable as it assigns different roles to players (survivors and killers).

The asymmetry between the roles results in interactions where a single action can have different effects on different players. For example, when a survivor fails a skill check. Other survivors perceive this as a loud noise and bright flash when nearby. However, the killer receives an extradiegetic indicator of the direction and distance. To classify this within the framework, the interaction was divided into two, survivor to survivor ( $\mathrm{S}$ to $\mathrm{S}$ ) and survivor to killer (S to K).

The $\mathrm{S}$ to $\mathrm{S}$ is a possible interaction that is mechanical and synchronous with always deducible awareness, always deducible feedback, possible initiator identification and an always identifiable recipient.

The $\mathrm{S}$ to $\mathrm{K}$ interaction differs in that it is guaranteed to be perceived by the killer. Thus the survivor can always deduce they've affected the killer. While the 
framework captured this asymmetry of perception by splitting the interaction, the relationship between the interactions is lost.

Asymmetry of agency is also a key aspect. The killer has the ability to significantly impact survivors, while survivors have little power against the killer. This is not reflected in our framework, in part due to the absence of roles, but also as there is no measurement of the impact of each interaction.

\section{Using the Framework for Multi-Participant Narratives}

To demonstrate the applicability of our framework to multi-participant narrative, we can consider a number of different narrative premises inspired by the different characteristics identified in the framework.

Premise 1. A spree of killings has recently occurred in London. Two detectives are working the case - one from the local police station, another a private investigator, hired by a relative of the victims. Each character is played by a different participant. The two will never meet, but their interactions with the crime scene and victims will change the course of the other's investigation.

This narrative is inspired by an interaction using possible deductive awareness and no feedback, with guaranteed interactions and no ability to identify initiator or recipient. The participants may deduce someone is interfering with their investigation, but will be unaware of the effect their actions have on the other. This creates two intertwined narratives but with notably different experiences.

Premise 2. The Research and Development department of a large corporation is on the verge of a new technological breakthrough. Participant one follows the head of this department who must oversee the final stages of the research. Participant two follows one of their employees, who unbeknownst to the head is a corporate spy whose job it is to steal and then sabotage the research.

This premise has clear asymmetry. The spy is always aware and always receives feedback on their attempts to undermine the department, while the head has only possible deductive awareness and possible deductive feedback, and will never identify the spy.

Premise 3. The first participant listens to the conversation of two women sitting in a bar. The older woman reminisces on the critical decisions and mistakes she has made in her life, interspersed with revelations about the younger women's life that are directed by the first participant, perhaps in reaction to the experiences of the older women. The younger women's story culminates in her having aged, finding herself back at the bar explaining her life story to a different younger woman. This telling of the life story is then used for the next participant.

This cyclic premise is inspired by an asynchronous interaction in which the initiator possibly receives deductive feedback, but as a recipient only has deductive awareness, as they are never told how their experience has been affected by the decisions of another participant, but might figure this out by reflecting on 
the decisions that they themselves have made. The other player is never identified. The time aspect element of asynchronous storytelling in this case facilitate time advancing at different rates for different participants, and means they can interact independently much as they would with a single participant narrative.

\section{Conclusion}

In this paper, we sought to describe the potential of multiplayer narratives by creating a framework of multiplayer game interactions, and looking at what narratives constructed using this framework might be like.

We did this by performing an iterative analysis of popular multiplayer video games in order to classify their different interactions and create a framework. We then validated the framework by applying it to three games notable for their novel multiplayer experiences. We then constructed a set of story premises based on these characteristics to demonstrate how the framework might inspire significantly differing types of multi-participant narrative.

The first of the questions we set out to answer was what are the core characteristics of interactions in games? In total our framework contains nine characteristics: Interaction Likelihood, Type, Synchronicity, Explicit Awareness, Deductive Awareness, Explicit Feedback, Deductive Feedback, Initiator Identifiability, and Recipient Identifiability. The prevalence of perception within the framework leaves open the question of whether providing the illusion of other players' agency on your story would be sufficient. Similar to how the illusion of agency can prove equally engaging to the real thing[17].

During validation, we found that the characteristics were capable of classifying all of the identified interactions within the selected games. However, the distinction between mechanical and informational interactions was less clear in Journey and Dead by Daylight. This suggests that Interaction Type is not binary, but rather more of a spectrum, making it harder to classify.

Similarly, we found that the framework missed something important when describing games where interactions varied between participants playing different roles, particularly in cases where the relative power of participants was noticeably different. Novel aspects of players' roles, goals and relationships may have been missed. These elements may be better studied at a game-wide level.

The second question we wanted to address was how we can use those characteristics to describe possible novel multi-participant narratives. In particular moving to a more concrete definition of the different sorts of experience that could be created. We have shown that the framework can describe a variety of different multi-participant narratives, and the characteristics derived from games can be equally applied to narratives. In particular the different combinations of Awareness, Feedback, and Identity, could result in subtly different experiences.

The work described here only starts to explore the potential of multi-participant narratives. With the framework giving a clear idea of the sorts of stories that are possible, we now intend to explore how they might be implemented and authored. For our future work, we intend to create a multi-participant narrative 
system based on a sculptural hypertext model[7][18], we have already begun to explore how this might work [8] and how it could support the wide range of interactions identified within the framework.

Our hope is that this framework will act as a foundation for future work in this area, allowing developers and researchers to understand the types of multiparticipant narratives that are possible, and build systems to support a variety of different multi-participant narrative experiences.

\section{References}

1. Cavazza, M., Charles, F., Mead, S.J.: Character-based interactive storytelling. IEEE Intelligent Systems 17(4), 17-24 (Jul 2002)

2. Ciarlini, A.E.M., Pozzer, C.T., Furtado, A.L., Feij, B.: A Logic-based Tool for Interactive Generation and Dramatization of Stories. In: ACE '05. pp. 133-140. ACM, New York, NY, USA (2005)

3. Mateas, M., Stern, A.: Facade: An experiment in building a fully-realized interactive drama. In: Game developers conference. vol. 2 (2003)

4. Riedl, M.O., Young, R.M.: An Intent-Driven Planner for Multi-Agent Story Generation. In: AAMAS '04. pp. 186-193. IEEE Computer Society, Washington, DC, USA (2004)

5. Fairclough, C., Cunningham, P.: A multiplayer case based story engine. Tech. rep., Trinity College Dublin, Department of Computer Science (2003)

6. Peinado, F., Gervs, P.: Transferring Game Mastering Laws to Interactive Digital Storytelling. In: SpringerLink, pp. 48-54. Springer Berlin Heidelberg, dOI: 10.1007/978-3-540-27797-2_7

7. Bernstein, M.: Card Shark and Thespis: Exotic Tools for Hypertext Narrative. In: ACM Hypertext '01. pp. 41-50. HYPERTEXT '01, ACM, New York, NY, USA (2001)

8. Spawforth, C., Millard, D.E.: Multiplayer Games as a Template for Multiplayer Narratives: A Case Study with Dark Souls. In: ACM Hypertext (2017)

9. Rocha, J.B., Mascarenhas, S., Prada, R.: Game mechanics for cooperative games. ZON Digital Games 2008 pp. 72-80 (2008)

10. Reuter, C., Wendel, V., Gbel, S., Steinmetz, R.: Game design patterns for collaborative player interactions. In: Proceedings of DiGRA (2014)

11. Cook, D.: What I've learned about designing multiplayer games so far, http://www.gamasutra.com/blogs/DanielCook/20140104/208021/What_Ive_ learned_about_designing_multiplayer_games_so_far.php

12. Zagal, J.P., Nussbaum, M., Rosas, R.: A model to support the design of multiplayer games. Presence: Teleoperators and Virtual Environments 9(5), 448-462 (2000)

13. Seif El-Nasr, M., Aghabeigi, B., Milam, D., Erfani, M., Lameman, B., Maygoli, H., Mah, S.: Understanding and Evaluating Cooperative Games. In: CHI '10. pp. 253-262. ACM, New York, NY, USA (2010)

14. Manninen, T.: Interaction forms in multiplayer desktop virtual reality games. In: VRIC2002 Conference. vol. 223, p. 232 (2002)

15. Manninen, T.: Rich Interaction in the Context of Networked Virtual Environments? Experiences Gained from the Multi-player Games Domain. In: People and Computers XV? Interaction without Frontiers, pp. 383-398. Springer London, London (2001), dOI: 10.1007/978-1-4471-0353-0_23 
16. Genette, G.: Narrative discourse: An essay in method. Cornell University Press (1983)

17. Fendt, M.W., Harrison, B., Ware, S.G., Cardona-Rivera, R.E., Roberts, D.L.: Achieving the Illusion of Agency. In: Interactive Storytelling. pp. 114-125. Springer, Berlin, Heidelberg (Nov 2012)

18. Millard, D.E., Hargood, C., Jewell, M.O., Weal, M.J.: Canyons, deltas and plains. ACM Hypertext '13 pp. 109-118 (2013) 